\title{
Developmental Manifestations of Grief in Children and Adolescents: Caregivers as Key Grief Facilitators
}

\author{
Lauren Alvis $^{1}$ (I) $\cdot$ Na Zhang $^{2} \cdot$ Irwin N. Sandler ${ }^{3}$ Julie B. Kaplow ${ }^{1}$ \\ Accepted: 22 December 2021 \\ (c) The Author(s), under exclusive licence to Springer Nature Switzerland AG 2021
}

\begin{abstract}
The death of a loved one represents one of the most distressing and potentially traumatic life events in childhood and adolescence. Grief reactions in youth are influenced by ongoing developmental processes and manifest differently depending on the child's age and developmental stage. These grief-related processes unfold within youths' caregiving context, as children and adolescents rely heavily on the adults in their environment to navigate and cope with the death of a loved one. Despite the field's increasing recognition of the potential for maladaptive grief reactions to impede functioning over time, few longitudinal research studies on childhood grief currently exist. In this article, we will (a) provide a brief overview of the childhood bereavement literature; (b) review the new DSM-5 and ICD-11 Prolonged Grief Disorder diagnostic criteria through a developmentally-informed lens; (c) describe how grief reactions manifest in children and adolescents of different ages through the lenses of multidimensional grief theory and relational developmental systems theory; (d) highlight key moderating factors that may influence grief in youth, and (e) discuss a primary moderating factor, the caregiving environment, and the potential mechanisms through which caregivers influence children's grief.
\end{abstract}

Keywords Bereavement $\cdot$ Childhood grief $\cdot$ Prolonged grief disorder $\cdot$ Parenting $\cdot$ Caregiving $\cdot$ Multidimensional grief theory $\cdot$ Relational developmental systems theory

The death of a caregiver, sibling, or loved one is one of the most commonly reported (Pynoos et al., 2014) and most distressing forms of trauma among youth (Kaplow et al., 2010). Due to the COVID-19 pandemic, rates of childhood bereavement, particularly among Black and Latino youth, are continuing to rise (Hillis et al., 2021). Childhood bereavement is associated with a range of mental and behavioral health problems, including depression (Cerel et al., 2006), posttraumatic stress reactions (Keyes et al., 2014), substance use (Kaplow et al., 2010), suicide-related behaviors (Guldin et al., 2015; Hill et al., 2019), decreased academic performance (Oosterhoff et al., 2018), and impairments in developmental tasks (Brent et al., 2012).

Lauren Alvis

lalvis@mmhpi.org

1 The Hackett Center for Mental Health, Meadows Mental Health Policy Institute, Houston, TX, USA

2 Human Development and Family Sciences, University of Connecticut, Storrs, CT 06269, USA

3 Department of Psychology, Arizona State University, Tempe, AZ 85281, USA
Few studies, however, have explicitly examined grief as an outcome among bereaved children and adolescents, in part due to a lack of consensus in the field with regard to the measurement of childhood grief.

Maladaptive grief reactions have been increasingly recognized, resulting in the recent inclusion of prolonged grief disorder (PGD) in the 11th edition of the International Classification of Diseases (ICD-11; WHO, 2018) and in Section II of the fifth Diagnostic and Statistical Manual for Psychiatric Disorders text revision (DSM-5-TR; APA, 2020). However, theory and research on PGD and maladaptive grief reactions more broadly have historically focused on adult grief or have assumed that childhood grief reactions and adult grief reactions are similar in nature. These traditional approaches to childhood grief fail to consider the unique ways that grief manifests as a function of the child's developmental stage at the time of loss (Kaplow et al., 2012) and often overlook a key factor known to affect child functioning following a death: the caregiving environment.

The primary aim of this article is to provide a developmentally-informed review of the state of the field with regard to childhood grief. We begin by providing a 
brief overview of the childhood bereavement literature and highlight the dearth of studies that have examined explicitly grief as an outcome. Next, we review the new DSM-5 and ICD-11 PDG diagnostic criteria through a developmentallyinformed lens. We then describe the phenomenology of childhood grief drawing from multidimensional grief theory and relational developmental systems theory, as well as key moderating factors that help to shape grief reactions among children and adolescents. Finally, we conclude with an overview of extant research on the critical role of a primary moderating factor-the caregiving environment- and describe potential mechanisms through which caregivers may facilitate adaptive coping and healthy grief reactions in their children following bereavement.

\section{Bereavement in Childhood and Adolescence}

In 2015, the United Nations International Children's Emergency Fund (UNICEF) estimated that worldwide nearly 140 million children under age 18 had experienced the death of one or both parents (UNICEF, 2017). In the US general population, $6.6 \%$ of children ( 4.5 million) will experience parental death and $1.5 \%$ will experience a sibling death before age 18 , and these rates are elevated in times of natural disasters, pandemics, and war (Burns et al., 2020). For example, a recent study estimated that over 140,000 youth in the U.S. have lost a parent or caregiver due to the COVID-19 pandemic, and that number continues to rise, particularly among youth of color (Hillis et al., 2021). Both sudden loss and witnessing death, are among the most prevalent types of trauma exposure among adolescents. For example, experiencing the sudden death of someone close was the most prevalent type of potentially traumatic experience $(30.2 \%)$, followed by witnessing the injury or death of someone $(12.9 \%)$ in a nationally representative sample of adolescents aged 13 to 18 (McChesney et al., 2015).

\section{Grief in Childhood and Adolescence}

A number of studies have examined links between childhood bereavement and problematic psychological and behavioral issues; however, very few have focused specifically on grief as an outcome. Although most bereaved children appear to grieve adaptively and maintain normal functioning across life domains (Kaplow et al., 2010; Keyes et al., 2014), research has shown that a subgroup of bereaved children experience "maladaptive grief" associated with impaired functioning, with estimates ranging from $10 \%$ in populationbased studies (Melhem et al., 2011) to $18 \%$ in youth referred for therapeutic services (Kaplow et al., 2018). Many studies of childhood grief suffer from several methodological limitations, including (1) the use of adult grief measures that lack developmentally-appropriate language for youth and do not assess the full range of grief reactions most often exhibited by children and adolescents; (2) lack of diversity within samples, both with regard to race/ethnicity or cause of death; and (3) lack of longitudinal studies that have examined grief reactions over time (Kaplow et al., 2014; Kaplow et al., 2019; Nader \& Layne, 2009).

\section{Current Diagnostic Criteria for Prolonged Grief Disorder}

The dearth of research on childhood grief may be due, in part, to a lack of consensus in the field regarding the conceptualization and accurate measurement of childhood grief, particularly maladaptive grief. To that end, the inclusion of Prolonged Grief Disorder (PGD) in the most recent edition of the ICD-11 and DSM-5-TR provides some "common language" to describe grief reactions that may be unhealthy or debilitating over time. Criteria for PGD as per the ICD-11 includes "persistent and pervasive longing for and/or persistent preoccupation with the deceased, accompanied by any of the 10 symptoms that indicate intense emotional pain (e.g., sadness, guilt, anger, denial, blame; difficulty accepting the death; feeling one has lost a part of one's self; an inability to experience positive mood; emotional numbness; difficulty in engaging with social or other activities) for at least six months following bereavement" (WHO, 2018). The ICD-11 includes a cultural caveat that specifies that grief reactions, in terms of the duration of symptoms as well as the expression and manifestation of grief, must clearly violate "expected social, cultural or religious norms for the individual's culture and context."

PGD in the DSM-5-TR (APA, 2020) can be diagnosed in children after at least 6 months have passed since the death of someone close (Criterion A), the child experiences intense yearning or preoccupation regarding the deceased person (Criterion B), accompanied by at least 3 of 8 symptoms of: identity disruption, disbelief about the death, avoidance (characterized by efforts to avoid reminders in children and adolescents), emotional pain related to the loss, difficulties moving on with life, emotional numbness, a sense that life is meaningless, and intense loneliness, nearly every day or more often, for at least one month (Criterion C), that cause distress or functional impairment (Criterion D), exceed cultural and contextual norms (Criterion E) and are not better explained by another mental disorder or substance (Criterion F; Prigerson et al., 2021).

Recent studies that have examined symptoms of PGD in children and adolescents offer support for the distinctiveness of PGD from other disorders, generally showing that 
symptoms of intense yearning, difficulties in accepting the loss, anger, and a sense that life is meaningless constitute a unique cluster of grief symptoms that can be distinguished from other symptoms that can occur post-loss, including PTSD and depression (Dillen et al., 2009; Spuij et al., 2012). Further, prolonged grief is significantly related to functional impairment in youth following the death of a loved one, even after accounting for PTSD and depression (Melhem et al., 2007; Spuji et al., 2012).

Despite the growing body of research on PGD, published studies of bereaved children have yet to systematically examine potential age-related manifestations or differences among DSM-5 or ICD-11 PGD symptom domains. PGD symptom presentations may be confounded by age due to youths' developing cognitive capacity and reliance on adults throughout the grieving process (Kentor \& Kaplow, 2020). For example, difficulties engaging in activities or moving on with life may manifest in youth as failure to achieve developmental milestones (Kaplow et al., 2012). Intense emotional pain in youth may be motivated by frustration and anger about feeling deprived of the deceased person's help in responding to their developmental needs, particularly when children lose a primary attachment figure (Layne et al., 2020). Moreover, anger may be exhibited behaviorally in young children as irritability, protest behavior, tantrums, oppositional behavior, and/or conduct problems, often in response to changes in daily routine or to others acting in the deceased person's role (Kaplow et al., 2012). In addition, experiencing disbelief about the death may not hold the same meaning in children under the age of 5 who lack the cognitive ability to understand the permanence of death compared with older children or adolescents who have more advance cognitive understandings of death (Sood et al., 2006). Taken together, the lack of developmentally-informed criteria for PGD can make it challenging for clinicians to accurately identify and diagnose PGD in children and adolescents (Layne et al., 2020).

\section{Conceptualizing Children's Grief through Multidimensional Grief Theory and Relational Developmental Systems Metatheory}

Although the diagnostic criteria for PGD are useful with regard to identifying grief reactions that may impede functioning in youth, additional theoretical work can help shed light on how and why children grieve in certain ways. Two of these theories are multidimensional grief theory, a developmentally-informed framework for understanding the broad range of adaptive and maladaptive grief reactions in youth (Kaplow et al., 2013; Layne et al., 2017), as well as relational developmental systems (RDS) metatheory, which proposes that developmental processes are dynamic and transactional and that adaptation is the result of reciprocal interactions between youth and their proximal (e.g., family, school) and distal (e.g., culture) ecologies (Lerner et al., 2018; Overton, 2015). RDS theory would suggest that changes in grief reactions can inform and are informed by cognitive, socioemotional, and identity developmental processes and that the ways in which bereavement-related problems manifest are likely to change over time as children move through various developmental stages and transitions (Kaplow et al., 2012; Kentor \& Kaplow, 2020). Further, an RDS metamodel emphasizes that all youth have the potential for positive adaptation in the aftermath of adversity (e.g., bereavement), particularly when their developmental needs and strengths align with contextual assets, such as a supportive caregiving environment (Lerner et al., 2018). Below, we review each domain of grief through the lenses of multidimensional grief theory and RDS metatheory and describe psychological and behavioral manifestations of grief as they relate to age-specific developmental processes.

Multidimensional grief theory proposes that childhood grief reactions can be characterized by three broad dimensions: Separation Distress, Existential/Identity Distress, and CircumstanceRelated Distress. The theory is based on the assumptions that both maladjustment and positive adjustment can manifest within each domain, and that positive and negative adjustment processes can and frequently do co-occur within a single domain (Kaplow et al., 2013; Layne et al., 2017).

Separation Distress centers on reactions to the continuing physical absence of, and the inability to physically reunite with the deceased person. Common initial manifestations of separation distress are typically characterized by missing the deceased person; heartache over his or her failure to return; and pining, yearning, and longing to be reunited with him/ her-often as evoked by loss reminders (Kaplow et al., 2013). These early feelings of separation distress can be adaptive by helping the child process and accept the reality of the loss and interact with others who may offer support (Wolfelt, 1996).

Youth struggling with separation distress may demonstrate developmental slowing or regression motivated by desires to stay connected with the deceased by remaining stuck in the same developmental stage, life circumstances, or immature/self-defeating behavior patterns one was in while they were still alive (Layne et al., 2017). In younger children this may involve behavioral regressions such as temper tantrums, increased irritability and/or language delays. Moreover, due to their budding understanding of the nature and permanence of death, toddlers or preschool age children may maintain expectations to see their deceased loved one again soon (e.g., waiting by the door for them to come home; Kaplow et al., 2012). Young school-aged children may express yearning in thought or play as a wish to literally physically reunite with the deceased (e.g., climb a ladder to heaven or use a toy 
phone to call their deceased loved one). In older children and adolescents, separation distress may sometimes take the form of a wish to die or fantasies of dying (i.e., suicidal ideation). These wishes reflect an intense desire to be reunited with their loved one and, depending on their own spiritual beliefs, may serve, in their own mind, as a means of overcoming the painful physical separation from their loved one (Kaplow et al., 2012; Kentor \& Kaplow, 2020). Developmental slowing may appear in adolescence as the avoidance of forming age-appropriate future aspirations, such as career and family goals (Kaplow et al., 2012; Layne et al., 2017).

Existential or Identity Distress refers to the altered sense of self (i.e., identity) and purpose (i.e., existential meaning one attributes to life) children and adolescents might experience after the death of a loved one, including feeling resigned to a seemingly ruined future (Layne et al., 2011). Maladaptive reactions to existential/identity-related challenges may manifest as severe disruptions in one's sense of self, or one's sense of purpose and meaning (e.g., life aspirations, future plans and ambitions).

Given younger children have difficulty articulating or grasping existential concepts such as the meaning of life, existential distress may manifest behaviorally in the form of lethargy, anhedonia, or social withdrawal (Kaplow et al., 2012). Young children may experience fears and concerns related to daily life (e.g., who is going to take me to school, brush my hair, help me with my homework, etc.). Both children and adolescents may express discontinuity in identity as shame or embarrassment surrounding the loss, as they may now feel different from others and subsequently selfconscious (e.g., I'm different from other kids because I don't have a mother anymore); however, this manifestation may be more salient in older children and adolescents due to their increased prioritization of peers combined with the critical developmental task of identity development during this time (Brown \& Larson, 2009; Kroger, 2006). Similarly, existential or identity distress can result in fears related to getting close to others or forming new relationships (e.g., I don't want to feel the pain of losing someone ever again) (Saltzman et al., 2017).Loss-related existential or identity crises may also manifest in adolescents as extreme risk-taking or recklessness, tempting fate, indifference to one's safety or well-being ("I don't care if I live or die"), and feeling like life is meaningless ("its's not worth trying" or "nothing really matters anymore"; Kaplow et al., 2012, 2013; Layne et al., 2017).

Circumstance-related Distress involves troubling thoughts and emotional pain over the particular manner of death and is theorized to increase in response to deaths that have occurred under tragic and potentially traumatic conditions (e.g., fatal accidents, homicide, suicide, neglect; Kaplow et al., 2014). Maladaptive responses to circumstancerelated distress are theorized to involve the encroachment of severe persisting distress reactions to the way the person died on adaptive grieving and mourning (Pynoos, 1992). Manifestations of this may include distressing mental images regarding the circumstances of the death, intense negative emotions (anger, rage, horror, revulsion, shame), distressing thoughts and beliefs regarding the deceased's manner of death, including blame of self and others, confusion, bewilderment, feeling shocked or dazed over how they died, and retaliatory fantasies (Layne et al., 2017).

Youth, particularly younger children, may receive less information to help them make sense of the circumstances of the death due to adults' assumptions about youths' limited capacity to understand. Failure to communicate clearly about the circumstances of the death may render children vulnerable to beliefs about possible culpability in causing or being unable to prevent the death, which may induce feelings of guilt and perpetuate distress (Cohen et al., 1977; Raveis, 1999). In addition, young children may re-enact distressing elements of the death through drawing or play, sometimes with alternate or counterfactual acts that depict what children feel they or others could have done to prevent the death (Kentor \& Kaplow, 2020). Due to adolescents' bourgeoning abstract thinking and perspective-taking abilities, they may experience negative emotions rooted in empathy and concern when thinking about the circumstances of their loved one's death (e.g., sadness over the suffering their loved one may have experienced).

Adaptive Grief Reactions the frequency, intensity, and distractibility of grief reactions are theorized to recede over time, often accompanied by an increasing capacity to access more affectively neutral or (in the case of healthy attachments and other supportive relationships) positive and comforting memories of the deceased (Kaplow et al., 2012). From an adaptive perspective, healthy manifestations of grief can facilitate healthy functioning, including a comforting connection to the deceased, honoring the deceased's memory, finding meaning, commitment to live a good life, and acts of service. For separation distress, this often involves finding helpful ways of feeling connected to the deceased individual or connected to other bereaved individuals who may be suffering. Adaptive responses to existential or identity distress can involve having a lifestyle that would have made the deceased person proud or carrying on their legacy in some way. Adaptive responses to circumstancerelated distress may consist of prosocial activities that aim to transform the circumstances of the death into something meaningful that can help others to avoid similar types of deaths - e.g., raising money for breast cancer if a loved one died of breast cancer (Kaplow et al., 2012, 2013; Saltzman et al., 2017). 


\section{Key Moderating Factors}

In considering the ways in which bereavement can have an impact on children's grief reactions, it is clear that numerous environmental and contextual factors can play critical roles in how children cope in the aftermath of a loved one's death. Although an extensive summary goes beyond the scope of this article, below we focus on some of the key contextual factors that have been found to contribute to youth's maladaptive grief reactions and the ways in which their impact may vary as a function of age/developmental stage.

\section{Circumstances of the Death}

The violent nature of certain circumstances surrounding a death, such as suicide or murder, can complicate children's grief reactions due to the likelihood of traumatic images associated with hearing about or witnessing the death (Pfeffer et al., 1997; Pynoos, 1992). Violent deaths have been associated with increased anxiety, depression, and maladaptive grief in adolescents (Dillen et al., 2009; Layne et al., 2008). However, some studies (e.g., Brown et al., 2007) report nonsignificant associations between the cause of the death and maladaptive grief reactions in youth. For example, Melhem et al. (2007) report that complicated grief scores of youth bereaved by a caregiver's suicide did not significantly differ from those bereaved by sudden natural deaths (e.g., heart attacks).

Studies of bereaved youth suggest the context of anticipated or illness-related losses, including terminal cancer, may be particularly distressing for children (Kaplow et al., 2020). For example, Kaplow and colleagues (2014) found that children who lost a caregiver due to a prolonged illness exhibited higher levels of both maladaptive grief and posttraumatic stress symptoms when compared to children who lost a caregiver due to sudden natural death (e.g., heart attack). The very nature of anticipated deaths may create more instances in which children are exposed to potentially traumatic elements (e.g., witnessing disturbing medical procedures and/or the dying person's progressive deterioration during repeated hospital visits) and accompanying intrusive preoccupations that may interfere with adaptive grief processes for children (Kaplow et al., 2014; Saldinger et al., 2004). Potentially traumatic elements of a death may be more subjective for children than for adults, given the child's less developed cognitive capacity to make sense of the observed physical changes taking place, the loss of security and/or safety associated with the death (particularly the loss of a primary caregiver), and their reliance on the caregiver to co-facilitate the grief process and co-create a narrative regarding the death itself (Kaplow et al., 2012; Lieberman et al., 2003).

\section{Time Since Loss}

Children and adolescents typically demonstrate a range of negative emotions and changes in behavior in the more immediate aftermath of a loved one's death, and the intensity of their grief reactions tend to diminish with time, usually 6-12 months after the loss (Maciejewski et al., 2007). Studies that have examined post-bereavement adjustment in youth over time suggest a subset may experience ongoing psychological or behavioral problems (e.g., Worden \& Silverman, 1996) or consistently severe maladaptive grief reactions two to three years post-loss (Schwartz et al., 2018). For example, Melhem et al (2011) found that $10.4 \%$ of parentally bereaved youth demonstrated persistently high maladaptive grief reactions over the course of 33 months after the death. In cross-sectional studies, findings regarding links between time elapsed since the death and grief reactions are mixed, with some finding an inverse association between time since loss and maladaptive grief (e.g., Alvis et al., 2020) and others finding non-signficant associations (e.g., Kaplow et al., 2014; Raveis et al., 1999).

\section{Relationship to the Deceased}

Another important circumstantial factor that can influence children's grief reactions is the relationship to the deceased person. The loss of "primary relationships" (e.g., parent) typically involves more intense psychological distress compared to "secondary" losses (e.g., extended family members, acquaintances) (Kaplow et al., 2010). In the context of a sniper attack, children who lost a primary attachment figure exhibited prolonged grief symptoms that were primarily characterized by separation distress, more so in comparison to youth who lost a friend or an extended family member (Holland \& Neimeyer, 2011). The nature of the relationship to the deceased is also important, as research shows relationships with the deceased that were close, supportive, and confiding are associated with an increased risk of maladaptive grief symptoms (Lobb et al., 2010). Additional research has found that adolescents who experienced the death of a friend had significantly higher maladaptive grief symptoms than those who experienced the death of a grandparent, and emotional closeness to the deceased was a positive predictor of maladaptive grief reactions regardless of the relationship to the person who died (Servaty-Seib \& Pistole, 2007). 


\section{Culture}

In the United States, research on grief has been largely based on the experiences of the dominant white culture (Laurie $\&$ Neimeyer, 2008). Although there has been a recent push for culturally-informed research on grief (e.g., Froyd et al., 2020), few empirical studies to date have examined culturally specific manifestations of grief and mourning in children and adolescents. Despite this fact, the new PGD criteria state "The duration of the bereavement reaction clearly exceeds expected social, cultural, or religious norms for the individual's culture and context."

Culture plays an important role in shaping youths' mourning rituals, communication about death, and socialization of emotion regulation strategies within families. For example, in European-American culture, parents often try to protect their children from hearing, thinking, or talking about death, believing young children are cognitively and emotionally incapable of handling death (Rosengren et al., 2014). In contrast, death is openly celebrated and embraced in Mexican culture, with Mexican children regularly exposed to symbolic images of death and actively participating in annual death rituals at a young age (Gutiérrez et al., 2020). Cultural practices like dia de los muertos provide socialization opportunities that influence youths' cognitive and affective understanding of death. However, it remains unclear how cultural practices that foster acceptance and openness about death early in life might contribute to potential differences in youths long term adjustment (i.e., grief reactions) following the loss of a loved one. There remains a critical need for more research that places culture at the forefront of understanding developmental differences in grief across childhood and adolescence.

\section{Race/Ethnicity}

When examining the bereavement experiences of youth of color, it is critical to situate those experiences within the systems of oppression (e.g., racism) that intersect with and shape the contexts in which they are exposed to and cope with bereavement. For example, in a study of clinic-referred children and adolescents, Black youth reported more severe maladaptive grief reactions compared to White youth, and this association was explained by an increased likelihood of experiencing the death of a loved one by homicide among Black youth (Douglas et al., 2021). A study of 1,581 bereaved college students at a large southern university also found that Black students reported higher levels of maladaptive grief symptoms than White students, especially when they spent less time speaking to others about their loss experience (Laurie \& Neimeyer, 2008). This may have been due, in part, to the fact that Black students in this sample also experienced more frequent bereavement by homicide as well as more intense grief reactions for the loss of extended kin beyond the immediate family (Laurie \& Neimeyer, 2008). In Black communities, the term "family" often extends beyond the traditional nuclear family to include kin who are not necessarily related by blood or marriage but held as closely as immediate family members (Nobles, 2004). While this extended network within Black communities lends itself to greater distress after the death of extended family members, it also provides critical support to families during times of adversity, such as bereavement. Black families are also more likely than other racial or ethnic groups to give and receive intergenerational support (Waites, 2009) and report a higher degree of religious and spiritual coping (Taylor et al., 2007), which may shape the ways in which children are socialized to grieve and understand death. For example, a greater focus on the afterlife in religious families may foster continuing bonds and help youth cope with separation distress.

\section{Caregiving Context}

Children and adolescents rely heavily on the adults in their environment to navigate and cope with the death of a loved one, making the caregiving context one of the most critical factors in facilitating adaptive grief (Kaplow et al., 2012; Alvis et al., 2020). The availability of a primary attachment figure in the aftermath of a death figure can help reduce fear and other negative emotions related to the loss, help reestablish normal routines, and serve as a biobehavioral regulator (Shear et al., 2007). Several aspects of the caregiving context that can have a promotive impact on adaptive grieving processes include effective social support, open communication, and positive parenting. Additionally, some studies highlight the importance of bereavementspecific behaviors in which caregiving can co-facilitate children's grief, such as reminiscing and talking about the deceased. In the next section, we discuss the role of these specific aspects of the caregiving environment on children's grief.

\section{Effects of the Caregiver Context on Grief and Adaptation of Bereaved Children}

\section{Relations of Domains of Parenting to Children's Grief}

We use Grusec and Davidov's (2010) domain-specific approach to child socialization to provide a framework for discussing the processes through which different aspects of caregiver behavior might impact children's grief. The framework identifies five domains of caregiver behavior protection, reciprocity, control, guided learning, and group participation - as distinct pathways to different socialization 
outcomes. Although the framework emphasizes specificity, linking each socialization domain to a specific developmental outcome via different processes, certain caregiver behaviors may reflect a combination or interplay of different domains of socialization (Grusec \& Davidov, 2010). We propose that several domains converge in explaining grief responses of bereaved children. More specifically, we propose that protection, reciprocity, and guided learning domains have particular relevance for understanding the effects of parenting on children's adaptive or maladaptive grief responses. In our review of the studies below, we describe specific domains of parenting followed by empirical findings supporting each domain. Finally, we suggest directions for future research to better understand the specific domains of parenting that may influence the development of maladaptive grief in bereaved youth.

The protection domain describes caregivers' sensitivity and responsiveness to their child's distress (Grusec \& Davidov, 2010). Bereaved children, particularly those who have experienced the death of a parent, have lost a major attachment figure, leading to distress over the loss (i.e., separation distress) as well as distress over their future security and safety within the family (i.e., existential distress). Wolchik et al. (2009) used a multi-component positive parenting construct to study the relations between parenting and children's grief. The positive parenting construct included multiple measures which appear to assess reassurance to the bereaved child, including instilling a sense of continuity of a loving, caring, and stable home environment. Specifically, the measures include: parental acceptance versus rejection (reversely scored), positive dyadic routines, parent's use of positive reinforcement, stable positive events in the family, child's feelings of being understood by their parent, and open communication. The construct was based on a second order confirmatory factor analysis of multiple child report, parent report and behavioral observation data from parent-child interaction tasks (Kwok et al., 2005) which had previously been found to discriminate bereaved children experiencing serious levels of mental health problems from those who did not experience serious mental health problems (Lin et al., 2004). As predicted, Wolchik et al. (2009) in a cross-sectional analysis found that the relation between positive parenting and children's maladaptive grief was mediated through the effects of positive parenting to decrease children's fears that there would be no one to take care of them in the future (labelled as fear of abandonment). They also found a significant direct prospective relation between positive parenting and intrusive grief thoughts 11-months later. In a similar vein, Grusec (2011) also proposed that the protection domain of parenting provides the sense of security that promotes children's effective emotional regulation, and a safe place to express their distress. Children's emotion regulation and emotion expressiveness may be another theoretical pathway through which the protective domain of parenting might decrease prolonged maladaptive grief responses in bereaved children.

There is also evidence that the protection domain of parenting contributes to positive adaptation for bereaved children. For example, post-traumatic growth is a positive adaptation outcome of trauma through forcing youth to reconsider their basic assumptions about life, who they are, and their relationships with others (Tedeschi \& Calhoun, 1996, 2004). Wolchik et al. (2009) found that youth-report of support seeking from parent and guardian prospectively predicted three aspects of growth through grief six years later, growth in relating to others, personal strengths and new possibilities. From the perspective of multidimensional grief theory, post-bereavement personal growth may represent positive adaptation to existential identity distress. And the protection domain of parenting may support bereaved youth' grief by facilitating growth and reducing maladaptive existential identity distress.

The reciprocity domain refers to the mutual responsiveness of parents to children and children to parents during daily interactions such as play (Grusec \& Davidov, 2010). A broadening of the original concept of reciprocity is parent-child dyadic mutuality in cooperative behavior and in acts of behavioral and emotional reciprocity (Grusec, 2011). One study in the literature may address this domain of parenting in relation to childhood grief. Shapiro et al. (2014) coded bereaved caregivers' interactions in a discussion with their bereaved children concerning positive memories of the deceased parent and the behaviors or characteristics that the child had in common with the deceased parent. They derived a measure of "parent communality" which consisted of a factor with high loadings on attunement and sensitivity to the child's needs, positive engagement, warmth and positivity, ease of conversation, and conversational depth. The parent communality factor was correlated with lower symptoms of children's maladaptive grief. Shapiro et al. (2014) also examined each individual parenting behavior and found that parent warmth and positivity was significantly related to lower maladaptive grief. The authors propose that parents' open, positive, engaged communication with their children about the loss may enable their children to ask questions and process the loss, leading to lower maladaptive grief symptoms.

The guided learning domain refers to scaffolding in which caregivers act as coaches to aid their child to accomplish tasks or learn skills (Grusec \& Davidov, 2010). This includes skills in emotion regulation, which is highly relevant to coping with grief (Shaver \& Tancredy, 2001). There is considerable research that caregivers play a critical role in the development of their child's emotion regulation skills. According to parental meta-emotion philosophy theory (Gottman et al., 1996), emotion coaching caregivers accept, 
validate, and empathize with their child's emotions, and provide tools to help the child to regulate emotions effectively, whereas emotion dismissing caregivers tend to minimize and invalidate their child's emotions, punishing the child for feeling negative emotions (Katz et al., 2012). For bereaved children, parental behavior involving discussions of children's grief are instances of emotion coaching or dismissing.

Alvis et al. (2020) developed a 36-item scale to assess caregiver's engagement in a range of grief-facilitating behaviors during the past month. In a large-scale study of 432 bereaved children and families, four factor analytic dimensions were derived from both the child report and parent report of grief facilitation behaviors: ongoing connection, existential continuity and support, caregiver grief expression, caregiver inhibition/avoidance of grief. The study found that child reports of their caregivers' avoidance and inhibition of grief discussions were related to higher levels of all three domains of maladaptive grief (i.e., separation distress, existential identity distress, and circumstances-related distress). The authors interpret the relation between inhibition/avoidance and higher levels of children's grief distress as possibly being due to caregiver avoidance increasing children's own inhibition of grief related emotion, which has previously been found to be associated with higher levels of child maladaptive grief, posttraumatic stress and depression symptoms (Dodd et al., 2020). There was also an unexpected relation between encouraging an ongoing connection with the deceased and higher levels of child maladaptive grief. The authors interpreted this as possibly due to children with more severe maladaptive grief reactions soliciting more ongoing connection from their surviving parents. There is also evidence that parent facilitation of grief is related to positive adaptation to the grief dimension of separation distress. Karydi (2018) in a retrospective study of adults who experienced the death of a parent during childhood found that parent facilitation of grief (e.g. through open discussions about the death, the deceased or expressing grief related feelings) was related to more positive continuing bonds with the deceased parent.

The group participation domain proposes that caregivers socialize their child by involving them in routines, rituals, or cultural practices (Grusec \& Davidov, 2010). Caregivers may adopt different kinds of familial or cultural practices to aid their child's grieving experiences, including involving children in attending the funeral and visiting the grave and commemorating loss anniversaries. Although several studies reported that retrospective memories of child sensitive involvement in mourning rituals (Saldinger et al., 2004) and children's satisfaction with their involvement in mourning practices (Sandler et al., 1988) were associated with lower child mental health problems, to our awareness no study has demonstrated a relation between parent facilitation of children's participation in mourning rituals and children's grief. Similarly, although studies have shown a relation between the control domain of parenting and child mental health problems (Sharp et al., 2020), we found no studies that demonstrated a relation between the control domain of parenting (i.e., use of discipline strategies) and children's grief responses.

Although there is empirical support for the association of protection, reciprocity, and guided learning domains of parenting behavior with children's grief responses, several critical questions remain for future research. For example, it is likely that parenting behaviors in the protection, reciprocity, and guided learning domains act in conjunction with one another (i.e., interplay of multiple domains; Grusec \& Davidov, 2011). It is also possible that measures of protection, reciprocity, and guided learning in previous studies only imperfectly represented each domain. Future research might develop measures that more specifically assess each of these domains to study their differential effects on children's grief. In particular, parenting in the guided learning (through sensitive discussions of grief) and protection (through providing a stable, caring and positive relationship with the caregiver) domains could have unique and additive effects on children's adaptive grief. Optimally, such studies would assess prospective longitudinal relations between domains of parenting to predict children's grief over time. Another critical question is whether there is specificity of effects of parenting domains on dimensions of children's grief or more general effects of parenting behavior on grief and child mental health problems. Several of the reviewed studies provide evidence for some aspects of parenting to relate to both grief dimensions and child mental health problems (e.g. Alvis et al., 2020; Shapiro et al., 2014). However, little is known about alternative prospective pathways in which parenting affects grief responses and mental health concurrently or whether there are cascading effects whereby parenting affects grief responses which in turn affect children's mental health over time. Deepened understanding about these questions will have implications for the design of interventions with caregivers that are effective and maximally efficient to reduce children's maladaptive grief response over time.

\section{Conclusions and Future Directions}

The recognition of prolonged grief disorder (PGD) as a mental health disorder in the ICD-11 and DSM-5-TR may spur research that continues to advance our understanding of childhood grief. However, the lack of developmentallyinformed diagnostic criteria make it difficult to accurately assess and diagnose PGD in children and adolescents - an issue which is further exacerbated by the dearth of research on culturally-specific manifestations of grief across development. The field will benefit by including 
developmentally- and culturally-informed measures that capture the full range of grief reactions theorized to manifest in children and adolescents (Kaplow et al., 2018). Longitudinal study designs using such measurement tools can provide evidence of the ways in which manifestations of grief change over time and can help to unpack developmental processes that dynamically intersect with and influence grief reactions in youth. These future studies should consider the various individual and contextual factors theorized to influence childhood grief (e.g., circumstances of the death, culture, race/ethnicity, and the caregiving environment) in order to elucidate potential mediating mechanisms. Specifically, given the importance of the caregiving environment following a death, studies that utilize measures of parenting behaviors that are specific to the context of bereavement (e.g., see Alvis et al., 2020) can help to further elucidate the ways in which caregivers can promote adaptive grieving and reduce maladaptive grieving in youth. These studies have the potential to inform preventive intervention efforts designed to reduce the risk of maladaptive grief and promote resilience among bereaved children and adolescents.

\section{Declarations}

Conflict of Interest On behalf of all authors, the corresponding author states that there is no conflict of interest.

\section{References}

Alvis, L. M., Dodd, C. G., Oosterhoff, B., Hill, R. M., Rolon-Arroyo, B., Logsdon, T., \& Kaplow, J. B. (2020). Caregiver behaviors and childhood maladaptive grief: Initial validation of the Grief Facilitation Inventory. Death Studies, 1-9.

American Psychiatric Association (APA). (2020). Board approves new prolonged grief disorder for DSM. Available at: gk7tr7 (Accessed 20 September 2021).

Brent, D. A., Melhem, N. M., Masten, A. S., Porta, G., \& Payne, M. W. (2012). Longitudinal effects of parental bereavement on adolescent developmental competence. Journal of Clinical Child \& Adolescent Psychology, 41(6), 778-791.

Brown, A. C., Sandler, I. N., Tein, J. Y., Liu, X., \& Haine, R. A. (2007). Implications of parental suicide and violent death for promotion of resilience of parentally-bereaved children. Death Studies, 31(4), 301-335.

Brown, B. B., \& Larson, J. (2009). Peer relationships in adolescence. In R. M. Lerner \& L. Steinberg (Eds.), Handbook of Adolescent Psychology (pp. 74-103). John Wiley \& Sons, Inc. https://doi.org/ 10.1002/9780470479193.adlpsy002004

Burns, M., Griese, B., King, S., \& Talmi, A. (2020). Childhood bereavement: Understanding prevalence and related adversity in the United States. American Journal of Orthopsychiatry, 90(4), 391-405. https://doi.org/10.1037/ort0000442

Cerel, J., Fristad, M. A., Verducci, J., Weller, R. A., \& Weller, E. B. (2006). Childhood bereavement: Psychopathology in the 2 years postparental death. Journal of the American Academy of Child \& Adolescent Psychiatry, 45(6), 681-690.
Cohen, P., Dizenhuz, I. M., \& Winget, C. (1977). Family adaptation to terminal illness and death of a parent. Social Casework, 58(4), 223-228.

Dillen, L., Fontaine, J. R. J., \& Verhofstadt-Denève, L. (2009). Confirming the distinctiveness of complicated grief from depression and anxiety among adolescents. Death Studies, 33(5), 437-461. https://doi.org/10.1080/07481180902805673

Dodd, C. G., Hill, R. M., Alvis, L. M., Rooney, E. E., Layne, C. M., Logsdon, T., Sandler, I. N., \& Kaplow, J. B. (2020). Initial validation and measurement invariance of the Active Inhibition Scale among traumatized and grieving youth. Journal of Traumatic Stress, 33(5), 843-849. https://doi.org/10.1002/jts.22529

Douglas, R. D., Alvis, L. M., Rooney, E. E., Busby, D. R., \& Kaplow, J. B. (2021). Racial, ethnic, and neighborhood income disparities in childhood posttraumatic stress and grief: Exploring indirect effects through trauma exposure and bereavement. Journal of Traumatic Stress, 34(5), 929-942. https://doi.org/10.1002/jts. 22732

Froyd, D., Jr., Robbins, B. D., Martinez, R., Benally, S., Begay, V., \& Roberts, C. (2020). Viewing trauma through a culturally informed framework: Tailoring interventions to culture. Journal of Family Trauma, Child Custody \& Child Development, 18(1), 41-65.

Gottman, J. M., Katz, L. F., \& Hooven, C. (1996). Parental metaemotion philosophy and the emotional life of families: Theoretical models and preliminary data. Journal of Family Psychology, $10(3), 243$.

Grusec, J. E. (2011). Socialization processes in the family: Social and emotional development. Annual Review of Psychology, 62, 243-269.

Grusec, J. E., \& Davidov, M. (2010). Integrating different perspectives on socialization theory and research: A domain-specific approach. Child Development, 81(3), 687-709.

Guldin, M.-B., Li, J., Pedersen, H. S., Obel, C., Agerbo, E., Gissler, M., Cnattingius, S., Olsen, J., \& Vestergaard, M. (2015). Incidence of suicide among persons who had a parent who died during their childhood: A population-based cohort study. JAMA Psychiatry, 72(12), 1227. https://doi.org/10.1001/jamapsychiatry. 2015.2094

Gutiérrez, I. T., Menendez, D., Jiang, M. J., Hernandez, I. G., Miller, P., \& Rosengren, K. S. (2020). Embracing death: Mexican parent and child perspectives on death. Child Development, 91(2). https://doi. org/10.1111/cdev.13263

Hill, R. M., Kaplow, J. B., Oosterhoff, B., \& Layne, C. M. (2019). Understanding grief reactions, thwarted belongingness, and suicide ideation in bereaved adolescents: Toward a unifying theory. Journal of Clinical Psychology, 75(4), 780-793.

Hillis, S. D., Blenkinsop, A., Villaveces, A., Annor, F. B., Liburd, L., Massetti, G. M., Unwin, H. J. T., et al. (2021). COVID-19associated orphanhood and caregiver death in the United States. Pediatrics. https://doi.org/10.1542/peds.2021-053760

Holland, J. M., \& Neimeyer, R. A. (2011). Separation and traumatic distress in prolonged grief: The role of cause of death and relationship to the deceased. Journal of Psychopathology and Behavioral Assessment, 33(2), 254-263. https://doi.org/10.1007/ s10862-010-9214-5

Kaplow, J. B., \& Layne, C. M. (2014). Sudden loss and psychiatric disorders across the life course: Toward a developmental lifespan theory of bereavement-related risk and resilience. American Journal of Psychiatry, 171(8), 807-810.

Kaplow, J. B., Layne, C., Oosterhoff, B., Goldenthal, H., Howell, K., Wamser-Nanney, R., \& Pynoos, R. (2018). Validation of the Persistent Complex Bereavement Disorder (PCBD) Checklist: A developmentally informed assessment tool for bereaved youth. Journal of Traumatic Stress, 31(2), 244-254.

Kaplow, J. B., Layne, C. M., Pynoos, R. S., Cohen, J., \& Lieberman, A. (2012). DSM-V diagnostic criteria for bereavement-related 
disorders in children and adolescents: Developmental considerations. Psychiatry, 75(3), 242-265.

Kaplow, J. B., Layne, C. M., Saltzman, W. R., Cozza, S. J., \& Pynoos, R. S. (2013). Using multidimensional grief theory to explore the effects of deployment, reintegration, and death on military youth and families. Clinical Child and Family Psychology Review, 16(3), 322-340.

Kaplow, J. B., Saunders, J., Angold, A., \& Costello, E. J. (2010). Psychiatric symptoms in bereaved versus non-bereaved youth and young adults: A longitudinal epidemiological study. Journal of the American Academy of Child and Adolescent Psychiatry, 49(11), 1145-1154. https://doi.org/10.1016/j.jaac.2010.08.004

Karydi, E. (2018). Childhood bereavement: The role of the surviving parent and the continuing bond with the deceased. Death Studies, 42(7), 415-425.

Katz, L. F., Maliken, A. C., \& Stettler, N. M. (2012). Parental metaemotion philosophy: A review of research and theoretical framework. Child Development Perspectives, 6(4), 417-422.

Kentor, R. A., \& Kaplow, J. B. (2020). Supporting children and adolescents following parental bereavement: Guidance for healthcare professionals. The Lancet Child \& Adolescent Health, 4(12), 889-898. https://doi.org/10.1016/S2352-4642(20) 30184-X

Keyes, K. M., Pratt, C., Galea, S., McLaughlin, K. A., Koenen, K. C., \& Shear, M. K. (2014). The burden of loss: Unexpected death of a loved one and psychiatric disorders across the life course in a national study. American Journal of Psychiatry, 171(8), 864-871. https://doi.org/10.1176/appi.ajp.2014.13081132

Kroger, J. (2006). Identity development: Adolescence through adulthood. Sage publications.

Kwok, O. M., Haine, R. A., Sandler, I. N., Ayers, T. S., Wolchik, S. A., \& Tein, J. Y. (2005). Positive parenting as a mediator of the relations between parental psychological distress and mental health problems of parentally bereaved children. Journal of Clinical Child and Adolescent Psychology, 34(2), 260-271.

Laurie, A., \& Neimeyer, R. A. (2008). African Americans in bereavement: Grief as a function of ethnicity. OMEGA - Journal of Death and Dying, 57(2), 173-193.

Layne, C. M., Kaplow, J. B., Oosterhoff, B., Hill, R. M., \& S Pynoos, R. (2017). The interplay between posttraumatic stress and grief reactions in traumatically bereaved adolescents: When trauma, bereavement, and adolescence converge. Adolescent Psychiatry, 7(4), 266-285. https://doi.org/10.2174/2210676608666180306162544

Layne, C. M., Olsen, J., Kaplow, J., \& Pynoos, R. (2011). Do traumatic circumstances of the death matter? Predicting the longitudinal course of grief in adolescents. In C. M. Layne (Chair), Developmental perspectives on proposed DSM-V bereavement criteria: Three longitudinal studies of bereaved children and adolescents. Symposium presented at the 27th Annual Meeting of the International Society for Traumatic Stress Studies, Baltimore, MD.

Layne, C. M., Oosterhoff, B., Pynoos, R. S., \& Kaplow, J. B. (2020). Developmental analysis of draft of DSM 5-TR criteria for Prolonged Grief Disorder: Report from the Child and Adolescent Bereavement Subgroup. Report submitted to the Panel on Developing Criteria for a Disorder of Pathological Grieving for DSM 5-TR (P. Applebaum, Chair). American Psychiatric Association.

Layne, C. M., Saltzman, W. R., Poppleton, L., Burlingame, G. M., Pašalić, A., Duraković, E., Mušić, M., Ćampara, N., Dapo, N., Arslanagić, B., Steinberg, A. M., \& Pynoos, R. S. (2008). Effectiveness of a school-based group psychotherapy program for warexposed adolescents: A randomized controlled trial. Journal of the American Academy of Child \& Adolescent Psychiatry, 47(9), 1048-1062.

Lerner, R. M., Brindis, C. D., Batanova, M., \& Blum, R. W. (2018). Adolescent health development: A relational developmental systems perspective. Handbook of Life Course Health Development, 109-121.

Lieberman, A. F., Compton, N. C., Van Horn, P., \& Ghosh Ippen, C. (2003). Losing a parent to death in the early years: Guidelines for the treatment of traumatic bereavement in infancy and early childhood. Zero to Three Press.

Lin, K. K., Sandler, I. N., Ayers, T. S., Wolchik, S. A., \& Luecken, L. J. (2004). Resilience in parentally bereaved children and adolescents seeking preventive services. Journal of Clinical Child and Adolescent Psychology, 33(4), 673-683.

Lobb, E. A., Kristjanson, L. J., Aoun, S. M., Monterosso, L., Halkett, G. K. B., \& Davies, A. (2010). Predictors of complicated grief: A systematic review of empirical studies. Death Studies, 34(8), 673-698. https://doi.org/10.1080/07481187.2010.496686

Maciejewski, P. K., Zhang, B., Block, S. D., \& Prigerson, H. G. (2007). An empirical examination of the Stage Theory of Grief. JAMA, 297(7), 716-723.

McChesney, G. C., Adamson, G., \& Shevlin, M. (2015). A latent class analysis of trauma based on a nationally representative sample of US adolescents. Social Psychiatry and Psychiatric Epidemiology, 50(8), 1207-1217. https://doi.org/10.1007/s00127-015-1075-5

Melhem, N. M., Moritz, G., Walker, M., Shear, M. K., \& Brent, D. (2007). Phenomenology and correlates of complicated grief in children and adolescents. Journal of the American Academy of Child \& Adolescent Psychiatry, 46(4), 493-499.

Melhem, N. M., Porta, G., Shamseddeen, W., Payne, M. W., \& Brent, D. A. (2011). Grief in children and adolescents bereaved by sudden parental death. Archives of General Psychiatry, 68(9), 911919. https://doi.org/10.1001/archgenpsychiatry.2011.101

Nader, K. O., \& Layne, C. M. (2009). Maladaptive grieving in children and adolescents: Discovering developmentally linked differences in the manifestation of grief. Stress Points, 23(5), 12-15.

Nobles, W. (2004). African philosophy: Foundation for Black psychology. In R. Jones (Ed.), Black psychology (4th ed., pp. 57-72). Cobb \& Henry Publishers.

Oosterhoff, B., Kaplow, J. B., \& Layne, C. M. (2018). Links between bereavement due to sudden death and academic functioning: Results from a nationally representative sample of adolescents. School Psychology Quarterly, 33(3), 372-380.

Overton, W. F. (2015). Process and relational developmental systems. In W. F. Overton, \& P. C. Molenaar (Eds.), Handbook of child psychology and developmental science. Vol. 1: Theory and method (7th ed.)

Pfeffer, C. R., Martins, P., Mann, J., Sunkenberg, M., Ice, A., Damore, J. P., Jr., \& Jiang, H. (1997). Child survivors of suicide: Psychosocial characteristics. Journal of the American Academy of Child \& Adolescent Psychiatry, 36(1), 65-74.

Prigerson, H. G., Boelen, P. A., Xu, J., Smith, K. V., \& Maciejewski, P. K. (2021). Validation of the new DSM-5-TR criteria for prolonged grief disorder and the PG-13-Revised (PG-13-R) scale. World Psychiatry, 20(1), 96-106.

Pynoos, R. S. (1992). Grief and trauma in children and adolescents. Bereavement Care, 11, 2-10.

Pynoos, R., Steinberg, A., Layne, C., Liang, L.-J., Vivrette, R., Briggs, E., Kisiel, C., Habib, M., Belin, T., \& Fairbank, J. (2014). Modeling constellations of trauma exposure in the National Child Traumatic Stress Network Core Data Set. Psychological Trauma Theory Research Practice and Policy, 6(51), S9-S17. https://doi. org/10.1037/a0037767

Raveis, V. H., Siegel, K., \& Karus, D. (1999). Children's psychological distress following the death of a parent. Journal of Youth and Adolescence, 28(2), 165-180.

Rosengren, K. S., Miller, P. J., Gutierrez, I. T., Chow, P. I., Schein, S. S., \& Anderson, K. N. (2014). Children's understanding of death: Toward a contextualized and integrated account. Monographs of the Society for Research in Child Development, 1(79), 70-77. 
Saldinger, A., Porterfield, K., \& Cain, A. C. (2004). Meeting the needs of parentally bereaved children: A framework for child-centered parenting. Psychiatry: Interpersonal and Biological Processes, 67(4), 331-352. https://doi.org/10.1521/psyc.67.4.331.56562

Saltzman, W., Layne, C., Pynoos, R., Olafson, E., Kaplow, J., \& Boat, B. (2017). Trauma and grief component therapy for adolescents: A modular approach to treating traumatized and bereaved youth. Cambridge University Press.

Sandler, I. N., Gersten, J. C., Reynold, D., Kallgren, C., \& Ramirez, R. (1988). Using theory and data to plan support intervention: Design of a program for bereaved children. In B. Gottlieb (Ed.), Marshalling Social Support: Formats, Processes and Effects (pp. 53-85). Sage.

Schwartz, L. E., Howell, K. H., \& Jamison, L. E. (2018). Effect of time since loss on grief, resilience, and depression among bereaved emerging adults. Death Studies, 42(9), 537-547. https://doi.org/ 10.1080/07481187.2018.1430082

Servaty-Seib, H. L., \& Pistole, M. C. (2007). Adolescent grief: Relationship category and emotional closeness. OMEGA - Journal of Death and Dying, 54(2), 147-167. https://doi.org/10.2190/ M002-1541-JP28-4673

Shapiro, D. N., Howell, K. H., \& Kaplow, J. B. (2014). Associations among mother-child communication quality, childhood maladaptive grief, and depressive symptoms. Death Studies, 38(3), 172-178.

Sharp, K. M. H., Meadows, E. A., Keim, M. C., Winning, A. M., Barrera, M., Gilmer, M. J., \& Gerhardt, C. A. (2020). The influence of parent distress and parenting on bereaved siblings' externalizing problems. Journal of Child and Family Studies, 29(4), 1081-1093.

Shaver, P. R., \& Tancredy, C. M. (2001). Emotion, attachment, and bereavement: A conceptual commentary. In M. S. Stroebe, R. O. Hansson, W. Stroebe, \& H. Schut (Eds.), Handbook of bereavement research: Consequences, coping, and care (pp. 63-88). American Psychological Association. https://doi.org/10.1037/ 10436-003

Shear, K., Monk, T., Houck, P., Melhem, N., Frank, E., Reynolds, C., \& Sillowash, R. (2007). An attachment-based model of complicated grief including the role of avoidance. European Archives of Psychiatry and Clinical Neuroscience, 257(8), 453-461. https://doi.org/10. 1007/s00406-007-0745-z
Sood, A. B., Razdan, A., Weller, E. B., \& Weller, R. A. (2006). Children's reactions to parental and sibling death. Current Psychiatry Reports, 8(2), 115-120.

Spuij, M., Reitz, E., Prinzie, P., Stikkelbroek, Y., de Roos, C., \& Boelen, P. A. (2012). Distinctiveness of symptoms of prolonged grief, depression, and post-traumatic stress in bereaved children and adolescents. European Child \& Adolescent Psychiatry, 21(12), 673-679. https://doi.org/10.1007/s00787-012-0307-4

Taylor, R. J., Chatters, L. M., \& Jackson, J. S. (2007). Religious and spiritual involvement among older African Americans, Caribbean Blacks, and non-Hispanic Whites: Findings from the national survey of American life. Journal of Gerontology Series B: Psychology and Scientific Social Science, 62(4), S238-S250.

Tedeschi, R. G., \& Calhoun, L. G. (1996). The Posttraumatic Growth Inventory: Measuring the positive legacy of trauma. Journal of Trauma Stress, 9, 455-471. https://doi.org/10.1007/BF02103658

Tedeschi, R. G., \& Calhoun, L. G. (2004). Posttraumatic growth: Conceptual foundations and empirical evidence. Psychological Inquiry, 15(1), 1-18.

United Nations International Children's Emergency Fund. (2017). Orphans. Retrieved May 16, 2018 from https://www.unicef.org/ media/media_45279.html

Waites, C. (2009). Building on strengths: Intergenerational practice with African American families. Social Work, 54(3), 278-287.

Wolchik, S. A., Coxe, S., Tein, J. Y., Sandler, I. N., \& Ayers, T. S. (2009). Six-year longitudinal predictors of posttraumatic growth in parentally bereaved adolescents and young adults. OMEGAJournal of Death and Dying, 58(2), 107-128.

Wolfelt, A. (1996). Healing the bereaved child. Companion Press.

Worden, J. W., \& Silverman, P. R. (1996). Parental death and the adjustment of school-age children. OMEGA - Journal of Death and Dying, 33(2), 91-102.

World Health Organization [WHO]. (2018). International Statistical Classification of Diseases and Related Health Problems. WHO.

Publisher's Note Springer Nature remains neutral with regard to jurisdictional claims in published maps and institutional affiliations. 